\title{
A NOTE ON UNIFORMLY DOMINATED SETS OF SUMMING OPERATORS
}

\author{
J. M. DELGADO and C. PIÑEIRO
}

Received 26 May 2001

\begin{abstract}
Let $Y$ be a Banach space that has no finite cotype and $p$ a real number satisfying $1 \leq p<\infty$. We prove that a set $\mu \subset \Pi_{p}(X, Y)$ is uniformly dominated if and only if there exists a constant $C>0$ such that, for every finite set $\left\{\left(x_{i}, T_{i}\right): i=1, \ldots, n\right\} \subset X \times \mathcal{M}$, there is an operator $T \in \Pi_{p}(X, Y)$ satisfying $\pi_{p}(T) \leq C$ and $\left\|T_{i} x_{i}\right\| \leq\left\|T x_{i}\right\|$ for $i=1, \ldots, n$.
\end{abstract}

2000 Mathematics Subject Classification: 47B10.

1. Introduction. Let $X$ and $Y$ be Banach spaces and $p$ a real number satisfying $1 \leq$ $p<\infty$. A subset $\mu$ of $\Pi_{p}(X, Y)$ is called uniformly dominated if there exists a positive Radon measure $\mu$ defined on the compact space $\left(B_{X^{*}},\left.\sigma\left(X^{*}, X\right)\right|_{B_{X^{*}}}\right)$ such that

$$
\|T x\|^{p} \leq \int_{B_{X^{*}}}\left|\left\langle x^{*}, x\right\rangle\right|^{p} d \mu\left(x^{*}\right)
$$

for all $x \in X$ and all $T \in \mathcal{M}$. Since the appearance of Grothendieck-Pietsch's domination theorem for $p$-summing operators, there is a great interest in finding out the structure of uniformly dominated sets. We will denote by $\mathscr{D}_{p}(\mu)$ the set of all operators $T \in$ $\Pi_{p}(X, Y)$ satisfying (1.1) for all $x \in X$. It is easy to prove that $\mathscr{D}_{p}(\mu)$ is absolutely convex, closed, and bounded (for the $p$-summing norm).

In [4], the authors consider the case $p=1$ and prove that $\mu \subset \Pi_{p}(X, Y)$ is uniformly dominated if and only if $\bigcup_{T \in \mathcal{M}} T^{*}\left(B_{Y^{*}}\right)$ lies in the range of a vector measure of bounded variation and valued in $X^{*}$.

In [3], the following sufficient condition is proved: "let $\mu \subset \Pi_{p}(X, Y)$ and $1 \leq p<$ $\infty$. Suppose that there is a positive constant $C>0$ such that, for every finite set $\left\{x_{1}, \ldots, x_{n}\right\}$ of $X$, there exists $Q \in \mathcal{M}$ satisfying $\pi_{p}(Q) \leq C$ and

$$
\sum_{i=1}^{n}\left\|T x_{i}\right\|^{p} \leq \sum_{i=1}^{n}\left\|Q x_{i}\right\|^{p}
$$

for all $T \in \mathcal{M}$. Then $\mathcal{M}$ is uniformly dominated." They also prove that this condition is necessary in the rather particular case that $\mathcal{M} \subset \Pi_{p}\left(c_{0}, c_{0}\right)$ and $\mathcal{M}=\mathscr{D}_{p}(\mu)$ for some positive Radon measure $\mu$ on $B_{\ell_{1}}$.

In this note, we obtain a necessary and sufficient condition for a set $\mu \subset \Pi_{p}(X, Y)$ to be uniformly dominated, with the only restriction that $Y$ is a Banach space without finite cotype. We refer to [1] for our operator terminology. If $X$ is a Banach space, $B_{X}$ will denote its closed unit ball; $\ell_{a}^{p}(X)\left(\ell_{w}^{p}(X)\right)$ will be the Banach space of the strongly (weakly) $p$-summable sequences. 
2. Main result. We need the following characterization of uniformly dominated sets.

Proposition 2.1. Let $1 \leq p<\infty$ and $\mu \subset \Pi_{p}(X, Y)$. The following statements are equivalent:

(a) $M$ is uniformly dominated.

(b) For every $\varepsilon>0$ and $\left(x_{n}\right) \in \ell_{w}^{p}(X)$, there exists $n_{0} \in \mathbb{N}$ such that

$$
\sum_{n \geq n_{0}}\left\|T_{n} x_{n}\right\|^{p}<\varepsilon
$$

for all sequences $\left(T_{n}\right)$ in $M$.

(c) There exists a constant $C>0$ such that

$$
\sum_{i=1}^{n}\left\|T_{i} x_{i}\right\|^{p} \leq C^{p} \sup _{x^{*} \in B_{X^{*}}} \sum_{i=1}^{n}\left|\left\langle x^{*}, x_{i}\right\rangle\right|^{p}
$$

for all $\left\{x_{1}, \ldots, x_{n}\right\} \subset X$ and $\left\{T_{1}, \ldots, T_{n}\right\} \subset \mathcal{M}$.

Proof. (a) $\Rightarrow$ (b). In a similar way as in the Pietsch factorization theorem [1], we can obtain, for all $T \in \mathcal{M}$, operators $U_{T}: L_{p}(\mu) \rightarrow \ell_{\infty}\left(B_{Y^{*}}\right),\left\|U_{T}\right\| \leq \mu\left(B_{X^{*}}\right)^{1 / p}$, and an operator $V: X \rightarrow L_{\infty}(\mu)$ such that the following diagram is commutative:

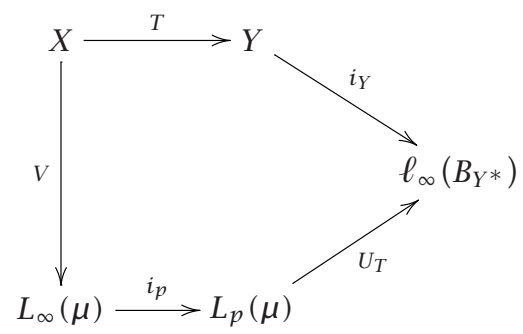

Here $i_{p}$ is the canonical injection from $L_{\infty}(\mu)$ into $L_{p}(\mu)$ and $i_{Y}$ is the isometry from $Y$ into $\ell_{\infty}\left(B_{Y^{*}}\right)$ defined by $i_{Y}(y)=\left(\left\langle y^{*}, y\right\rangle\right)_{y^{*} \in B_{Y^{*}}}$. Given $\varepsilon>0$ and $\left(x_{n}\right) \in \ell_{w}^{p}(X)$, we can choose $n_{0} \in \mathbb{N}$ so that

$$
\sum_{n \geq n_{0}}\left\|i_{p} \circ V\left(x_{n}\right)\right\|^{p}<\frac{\varepsilon}{\mu\left(B_{X^{*}}\right)}
$$

because $i_{p} \circ V$ is $p$-summing. Then, if $\left(T_{n}\right)$ is a sequence in $M$, we have

$$
\begin{aligned}
\sum_{n \geq n_{0}}\left\|T_{n} x_{n}\right\|^{p} & =\sum_{n \geq n_{0}}\left\|i_{Y} \circ T_{n}\left(x_{n}\right)\right\|^{p} \\
& =\sum_{n \geq n_{0}}\left\|U_{T_{n}} \circ i_{p} \circ V\left(x_{n}\right)\right\|^{p} \\
& \leq \mu\left(B_{X^{*}}\right) \sum_{n \geq n_{0}}\left\|i_{p} \circ V\left(x_{n}\right)\right\|^{p} \leq \varepsilon .
\end{aligned}
$$

(b) $\Rightarrow$ (c). Using a standard argument, we can prove that $\mathcal{M}$ is bounded for the operator norm. Hence, given $\hat{x}=\left(x_{n}\right) \in \ell_{w}^{p}(X)$, there exists $M_{\hat{x}}>0$ such that

$$
\sum_{n=1}^{\infty}\left\|T_{n} x_{n}\right\|^{p} \leq M_{\hat{x}}
$$


for all $\left(T_{n}\right)$ in $M$. Then, we can consider the linear maps

$$
\widehat{T}:\left(x_{n}\right) \in \ell_{w}^{p}(X) \longmapsto\left(T_{n} x_{n}\right) \in \ell_{a}^{p}(Y)
$$

for each $\hat{T}=\left(T_{n}\right)$ in $M$. They have closed graph; so, by the uniform boundedness principle, there exists $M>0$ so that

$$
\left(\sum_{n=1}^{\infty}\left\|T_{n} x_{n}\right\|^{p}\right)^{1 / p} \leq M \epsilon_{p}\left(x_{n}\right)
$$

for all $\left(x_{n}\right) \in \ell_{w}^{p}(X)$ and all $\left(T_{n}\right)$ in $\mathcal{M}$ (we wrote $\epsilon_{p}$ for the norm in $\ell_{w}^{p}(X)$ ).

(c) $\Rightarrow$ (a). Given $A=\left\{T_{1}, \ldots, T_{n}\right\} \subset \mathcal{M}$ and $B=\left\{x_{1}, \ldots, x_{n}\right\} \subset X$, we define $f_{A, B}: B_{X^{*}} \rightarrow \mathbb{R}$ by

$$
f_{A, B}\left(x^{*}\right)=C^{p}\left(\sum_{i=1}^{n}\left|\left\langle x^{*}, x_{i}\right\rangle\right|^{p}\right)-\sum_{i=1}^{n}\left\|T_{i} x_{i}\right\|^{p}
$$

for all $x^{*} \in X^{*}$. We denote by $\mathscr{P}$ the set of all functions $f_{A, B}$. It is clear that $\mathscr{P}$ is convex and disjoint from the cone $\mathcal{N}=\left\{f \in \mathscr{C}\left(B_{X^{*}}\right): f\left(x^{*}\right)<0\right.$, for all $\left.x^{*} \in B_{X^{*}}\right\}$. In a similar way as in the proof of Pietsch's domination theorem [1], we can show that there is a probability measure $\mu$ on $B_{X^{*}}$ satisfying

$$
\int_{B_{X^{*}}}\left(\|T x\|^{p}-C^{p}\left|\left\langle x^{*}, x\right\rangle\right|^{p}\right) d \mu \leq 0
$$

for all $T \in \mathcal{M}$ and all $x \in X$.

As an application of this result, we can show a relatively compact set for the $p$ summing norm which is not uniformly dominated. Put $T_{n}=(1 / n) e_{n}^{*} \otimes e_{n}, n \in \mathbb{N}$, where $\left(e_{n}\right)$ and $\left(e_{n}^{*}\right)$ are the unit basis of $c_{0}$ and $\ell_{1}$, respectively. As $\pi_{1}\left(T_{n}\right)=1 / n$, $\left(T_{n}\right)$ is a null sequence in $\Pi_{1}\left(c_{0}, c_{0}\right)$, so $\left(T_{n}\right)$ is relatively compact. To see that it is not uniformly dominated, we will use Proposition 2.1: the sequence $\left(e_{n}\right)$ is weakly summable but, for all $n \in \mathbb{N}$, we have

$$
\sum_{k \geq n}\left\|T_{k} e_{k}\right\|_{\infty}=\sum_{k \geq n} \frac{1}{k} .
$$

We are now ready to introduce our main result.

THEOREM 2.2. Let $Y$ be a Banach space that has no finite cotype, $\mu \subset \Pi_{p}(X, Y)$, and $1 \leq p<\infty$. The following statements are equivalent:

(a) $M$ is uniformly dominated.

(b) There is a constant $C>0$ such that, for every $\left\{x_{1}, \ldots, x_{n}\right\} \subset X$ and $\left\{T_{1}, \ldots, T_{n}\right\} \subset \mathcal{M}$, there exists an operator $T \in \Pi_{p}(X, Y)$ satisfying $\pi_{p}(T) \leq C$ and

$$
\left\|T_{i} x_{i}\right\| \leq\left\|T x_{i}\right\|, \quad i=1, \ldots, n .
$$

Proof. (a) $\Rightarrow(b)$. By hypothesis, there exists a positive Radon measure $\mu$ on $B_{X^{*}}$ such that

$$
\|T x\| \leq\left(\int_{B_{X^{*}}}\left|\left\langle x^{*}, x\right\rangle\right|^{p} d \mu\left(x^{*}\right)\right)^{1 / p}
$$


for all $T \in \mathcal{M}$ and all $x \in X$. Since $Y$ has no finite cotype, $Y$ contains $\ell_{\infty}^{n}$ 's uniformly. By [2], for every $\varepsilon>0$ and $n \in \mathbb{N}$, there is an isomorphism $J_{n}$ from $\ell_{\infty}^{n}$ onto a subspace of $Y$ satisfying $\left\|J_{n}^{-1}\right\|=1$ and $\left\|J_{n}\right\| \leq 1+\varepsilon$ for all $n \in \mathbb{N}$.

Given $\left\{x_{1}, \ldots, x_{n}\right\} \subset X$ and $\left\{T_{1}, \ldots, T_{n}\right\} \subset \mathcal{M}$, by (2.13) we have

$$
\left\|T_{i} x_{i}\right\| \leq\left(\int_{B_{X^{*}}}\left|\left\langle x^{*}, x_{i}\right\rangle\right|^{p} d \mu\left(x^{*}\right)\right)^{1 / p}, \quad i=1, \ldots, n .
$$

For every $i=1, \ldots, n$, take $g_{i} \in L_{q}(\mu)$ such that $\left\|g_{i}\right\|_{q}=1$ and

$$
\left(\int_{B_{X^{*}}}\left|\left\langle x^{*}, x_{i}\right\rangle\right|^{p} d \mu\left(x^{*}\right)\right)^{1 / p}=\int_{B_{X^{*}}}\left\langle x^{*}, x_{i}\right\rangle g_{i}\left(x^{*}\right) d \mu\left(x^{*}\right) .
$$

From (2.14) and (2.15), we obtain

$$
\left\|T_{i} x_{i}\right\| \leq \int_{B_{X^{*}}}\left\langle x^{*}, x_{i}\right\rangle g_{i}\left(x^{*}\right) d \mu\left(x^{*}\right), \quad i=1, \ldots, n .
$$

Put $y_{i}=J_{n} e_{i}$, being $\left(e_{i}\right)_{i=1}^{n}$ the unit basis of $\ell_{\infty}^{n}$. We define an operator $T: X \rightarrow Y$ by

$$
T x=\sum_{i=1}^{n}\left(\int_{B_{X^{*}}}\left\langle x^{*}, x\right\rangle g_{i}\left(x^{*}\right) d \mu\left(x^{*}\right)\right) y_{i} .
$$

We first prove that $\|T x\|^{p} \leq\left(\int_{B_{X^{*}}}\left|\left\langle x^{*}, x\right\rangle\right|^{p} d \mu\left(x^{*}\right)\right)(1+\varepsilon)$ for all $x \in X$ :

$$
\begin{aligned}
\|T x\| & =\sup _{y^{*} \in B_{Y^{*}}}\left|\left\langle y^{*}, \sum_{i=1}^{n}\left(\int_{B_{X^{*}}}\left\langle x^{*}, x\right\rangle g_{i}\left(x^{*}\right) d \mu\left(x^{*}\right)\right) y_{i}\right\rangle\right| \\
& \leq \sup _{y^{*} \in B_{Y^{*}}} \sum_{i=1}^{n}\left(\int_{B_{X^{*}}}\left|\left\langle x^{*}, x\right\rangle\right|\left|g_{i}\left(x^{*}\right)\right| d \mu\left(x^{*}\right)\right)\left|\left\langle y^{*}, y_{i}\right\rangle\right| \\
& \leq \sup _{y^{*} \in B_{Y^{*}}} \sum_{i=1}^{n}\left(\int_{B_{X^{*}}}\left|\left\langle x^{*}, x\right\rangle\right|^{p} d \mu\left(x^{*}\right)\right)^{1 / p}\left(\int_{B_{X^{*}}}\left|g_{i}\left(x^{*}\right)\right|^{q} d \mu\left(x^{*}\right)\right)^{1 / q}\left|\left\langle y^{*}, y_{i}\right\rangle\right| \\
& \leq\left(\int_{B_{X^{*}}}\left|\left\langle x^{*}, x\right\rangle\right|^{p} d \mu\left(x^{*}\right)\right)^{1 / p} \sup _{y^{*} \in B_{Y^{*}}} \sum_{i=1}^{n}\left|\left\langle y^{*}, y_{i}\right\rangle\right| \\
& \leq\left(\int_{B_{X^{*}}}\left|\left\langle x^{*}, x\right\rangle\right|^{p} d \mu\left(x^{*}\right)\right)^{1 / p}\left\|J_{n}^{*}\right\| \\
& \leq\left(\int_{B_{X^{*}}}\left|\left\langle x^{*}, x\right\rangle\right|^{p} d \mu\left(x^{*}\right)\right)^{1 / p}(1+\varepsilon) .
\end{aligned}
$$

Finally, we need to prove that $\left\|T_{i} x_{i}\right\| \leq\left\|T x_{i}\right\|$ for $i=1, \ldots, n$. Put $y_{i}^{*}=e_{i}^{*} \circ J_{n}^{-1}$, $\left(e_{i}^{*}\right)_{i=1}^{n}$ being the unit basis of $\left(\ell_{\infty}^{n}\right)^{*} \simeq \ell_{1}^{n}$. Notice that $\left\|y_{i}^{*}\right\| \leq 1$ for $i=1, \ldots, n$. We 
also denote by $y_{i}^{*}$ a Hahn-Banach extension of $e_{i}^{*} \circ J_{n}^{-1}$ to $Y$. We have

$$
\begin{aligned}
\left\|T x_{i}\right\| & \geq\left|\left\langle y_{i}^{*}, T x_{i}\right\rangle\right| \\
& =\left|\left\langle y_{i}^{*}, \sum_{j=1}^{n}\left(\int_{B_{X^{*}}}\left\langle x^{*}, x_{i}\right\rangle g_{j}\left(x^{*}\right) d \mu\left(x^{*}\right)\right) y_{j}\right\rangle\right| \\
& =\left|\sum_{j=1}^{n}\left(\int_{B_{X^{*}}}\left\langle x^{*}, x_{i}\right\rangle g_{j}\left(x^{*}\right) d \mu\left(x^{*}\right)\right)\left\langle y_{i}^{*}, y_{j}\right\rangle\right| \\
& =\left|\sum_{j=1}^{n}\left(\int_{B_{X^{*}}}\left\langle x^{*}, x_{i}\right\rangle g_{j}\left(x^{*}\right) d \mu\left(x^{*}\right)\right)\left\langle e_{i}^{*} \circ J_{n}^{-1}, J_{n} e_{j}\right\rangle\right| \\
& =\left|\sum_{j=1}^{n}\left(\int_{B_{X^{*}}}\left\langle x^{*}, x_{i}\right\rangle g_{j}\left(x^{*}\right) d \mu\left(x^{*}\right)\right)\left\langle e_{i}^{*}, e_{j}\right\rangle\right| \\
& =\int_{B_{X^{*}}}\left\langle x^{*}, x_{i}\right\rangle g_{i}\left(x^{*}\right) d \mu\left(x^{*}\right) \\
& \geq\left\|T_{i} x_{i}\right\|,
\end{aligned}
$$

the last inequality is due to (2.16).

(b) $\Rightarrow$ (a). It follows easily using Proposition 2.1(c).

REMARKS. (1) It is interesting to give an example of a uniformly dominated set $\mu$ for which there is no operator $T \in \mathcal{M}$ satisfying $\left\|T_{i} x_{i}\right\| \leq\left\|T x_{i}\right\|, i=1, \ldots, n$, for some finite set $\left\{\left(x_{i}, T_{i}\right): i=1, \ldots, n\right\} \subset X \times \mathcal{M}$. Let $X=\ell_{1}$ and $Y=\ell_{\infty}$ and consider the set $M=\left\{T_{\beta}: \beta \in B_{\ell_{2}}\right\}, T_{\beta}: \ell_{1} \rightarrow \ell_{\infty}$ being defined by $T_{\beta}(\alpha)=\left(\alpha_{n} \beta_{n}\right)$ for all $\alpha=\left(\alpha_{n}\right) \in \ell_{1}$. Obviously, $M$ is a uniformly dominated subset of $\Pi_{1}\left(\ell_{1}, \ell_{\infty}\right)$.

By contradiction, suppose the following condition holds: "there is a constant $C>0$ such that, for every finite set $\left\{\left(x_{i}, T_{i}\right): i=1, \ldots, n\right\} \subset X \times \mathcal{M}$, there exists $T \in \mathcal{M}$ satisfying $\left\|T_{i} x_{i}\right\| \leq C\left\|T x_{i}\right\|, i=1, \ldots, n$." Put $x_{i}=e_{i}$ and $T_{i}=T_{\beta_{i}}$ for $i=1, \ldots, n$, where $\left(e_{i}\right)_{i=1}^{\infty}$ is the unit basis of $\ell_{1}$ and $\beta_{i}=(1 / \sqrt{i},(i), 1 / \sqrt{i}, 0, \ldots)$. Take $T_{\gamma} \in \mathcal{M}$ such that

$$
\left\|T_{i} x_{i}\right\| \leq C\left\|T_{\gamma} x_{i}\right\|, \quad i=1, \ldots, n ;
$$

this yields

$$
\frac{1}{\sqrt{i}} \leq C\left|\gamma_{i}\right|, \quad i=1, \ldots, n
$$

Then we have

$$
1 \geq \sum_{i=1}^{\infty}\left|\gamma_{i}\right|^{2} \geq \sum_{i=1}^{n}\left|\gamma_{i}\right|^{2} \geq \frac{1}{C^{2}} \sum_{i=1}^{n} \frac{1}{i} .
$$

So, we have obtained the inequality $\sum_{i=1}^{n} 1 / i \leq C^{2}$ for all $n \in \mathbb{N}$ which allows us to state that such an operator $T$ cannot exist.

(2) Notice that, in the above example, $\boldsymbol{M}$ is absolutely convex and weakly compact in $\Pi_{1}\left(\ell_{1}, \ell_{\infty}\right)$. Then, $\mathcal{M}$ is absolutely convex, closed, and uniformly dominated but $\mu \neq \mathscr{D}_{1}(\mu)$ for every admissible positive Radon measure $\mu$. 
(3) Finally, we give an example of a bounded set $\mu$ of 2-summing operators that does not have property (b) in Theorem 2.2. Consider the set $\mathcal{M}$ of all 2-summing operators $T_{\beta}: c_{0} \rightarrow \ell_{\infty}$ defined by $T_{\beta}(\alpha)=\left(\alpha_{n} \beta_{n}\right)$ for all $\alpha=\left(\alpha_{n}\right) \in c_{0}$, where $\beta=\left(\beta_{n}\right)$ runs over the unit ball of $\ell_{2}$. We have $T_{\beta}=i \circ S_{\beta}$, $i$ being the identity map from $\ell_{2}$ into $\ell_{\infty}$ and $S_{\beta}: c_{0} \rightarrow \ell_{2}$ defined by $S_{\beta}(\alpha)=\left(\alpha_{n} \beta_{n}\right)$. Since $\ell_{2}$ has cotype 2 , it follows that $S_{\beta}$ is 2 -summing [1]. Nevertheless, $\mathcal{M}$ does not satisfy property (b) in the above theorem. By contradiction, suppose that there is a constant $C>0$ such that (b) holds. Again, we take $\tilde{\beta}_{i}=(1 / \sqrt{i},(i), 1 / \sqrt{i}, 0, \ldots)$ for all $i \in \mathbb{N}$. By hypothesis, there exists $T \in \Pi_{2}\left(c_{0}, \ell_{\infty}\right)$ such that $\pi_{2}(T) \leq C$ and $\left\|T_{\tilde{\beta}_{i}} e_{i}\right\| \leq\left\|T e_{i}\right\|$ for $i=1, \ldots, n$. Then we have

$$
\sum_{i=1}^{n} \frac{1}{i}=\sum_{i=1}^{n}\left\|T_{\tilde{\beta}_{i}} e_{i}\right\|^{2} \leq \sum_{i=1}^{n}\left\|T e_{i}\right\|^{2} \leq C^{2}
$$

for all $n \in \mathbb{N}$. Hence, $M$ does not have property (b) in Theorem 2.2.

\section{REFERENCES}

[1] J. Diestel, H. Jarchow, and A. Tonge, Absolutely Summing Operators, Cambridge Studies in Advanced Mathematics, vol. 43, Cambridge University Press, Cambridge, 1995.

[2] R. C. James, Uniformly non-square Banach spaces, Ann. of Math. (2) 80 (1964), 542-550.

[3] R. Khalil and M. Hussain, Uniformly dominated sets of p-summing operators, Far East J. Math. Sci. (1998), Special Volume, Part I, 59-68.

[4] B. Marchena and C. Piñeiro, Bounded sets in the range of an $X^{* *}$-valued measure with bounded variation, Int. J. Math. Math. Sci. 23 (2000), no. 1, 21-30.

J. M. Delgado: Departamento de Matemáticas, Escuela Politécnica SuPerior, UniVerSIDAD DE HuElVA, LA RÁBIDA 21819, HuELVA, SPAIN

E-mail address: jmde1ga@uhu.es

C. Piñeiro: Departamento de Matemáticas, Escuela Politécnica Superior, UniversiDAD DE Huelva, La RÁbIdA 21819, HuelVA, SPAIN

E-mail address: candido@uhu.es 


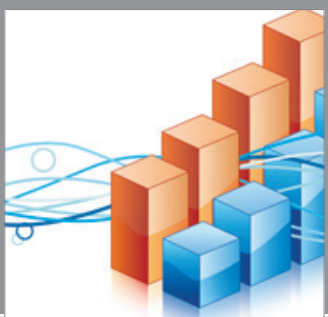

Advances in

Operations Research

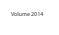

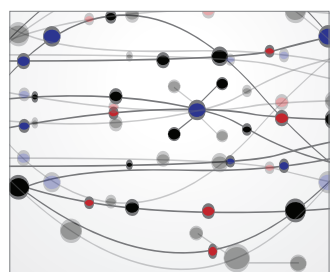

\section{The Scientific} World Journal
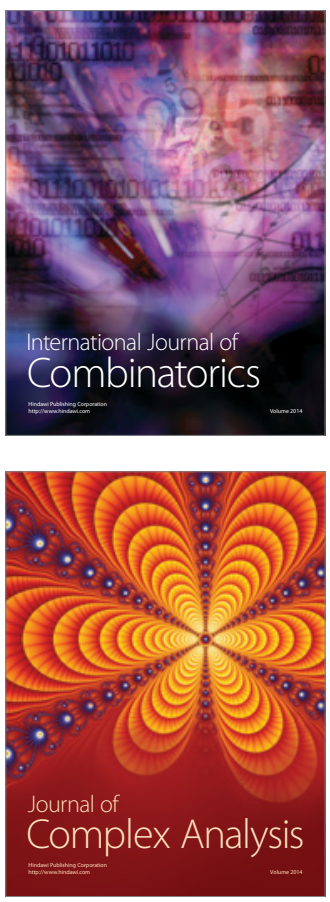

International Journal of

Mathematics and

Mathematical

Sciences
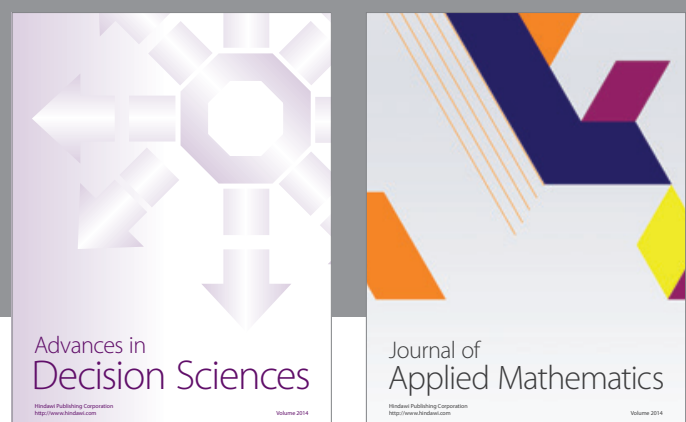

Journal of

Applied Mathematics
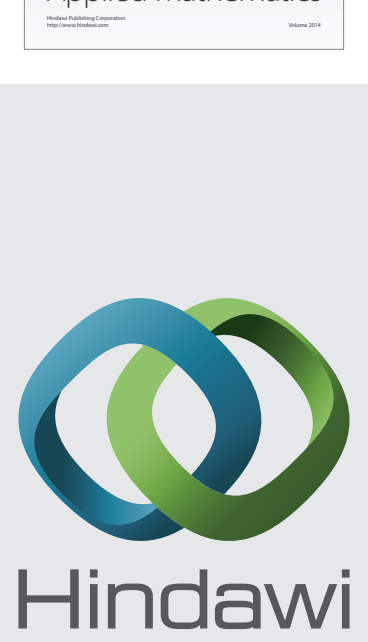

Submit your manuscripts at http://www.hindawi.com
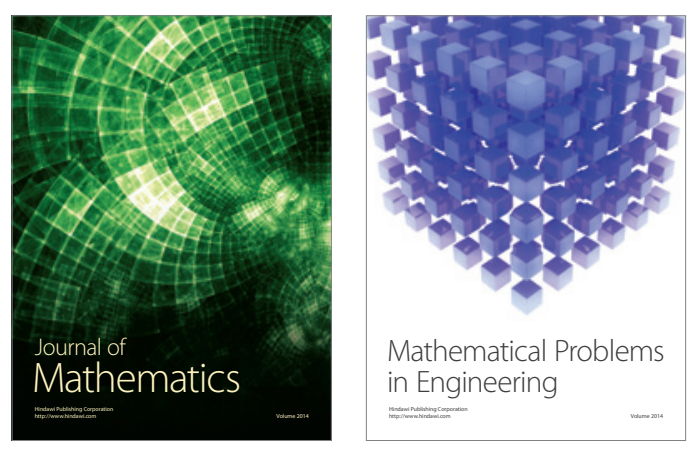

Mathematical Problems in Engineering
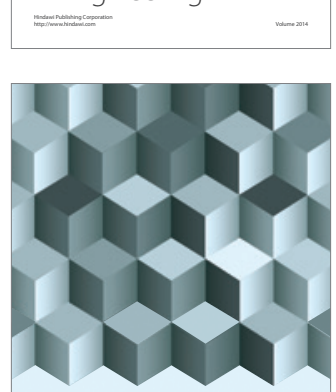

Journal of

Function Spaces
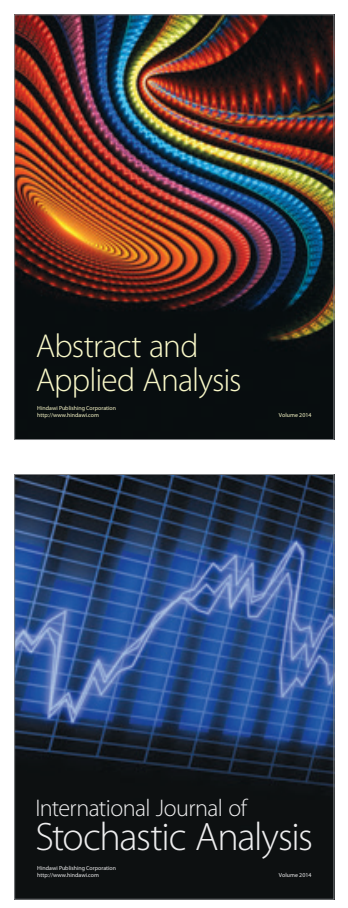

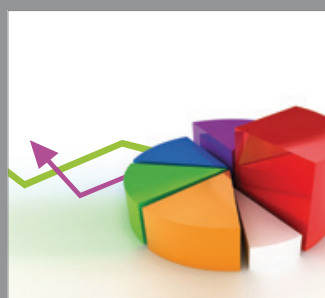

ournal of

Probability and Statistics

Promensencen
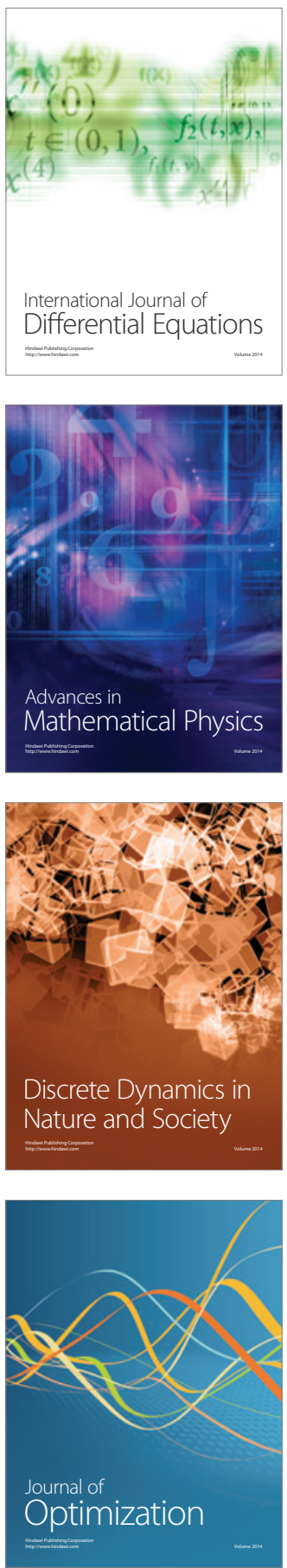